\title{
Metodologias de Ensino de Matemática na Robótica Educacional: um mapeamento sistemático
}

\author{
Neumar Regiane Machado Albertoni - UTFPR/Curitiba - \\ neumarmatematica@gmail.com \\ Giane Fernanda Schneider Gross - UTFPR/Curitiba - giane.fer@gmail.com \\ Érica Oliveira dos Santos - UTFPR/Curitiba - ericaoliveirasantos88@gmail.com \\ Marco Aurélio Kalinke - UTFPR/Curitiba - marcokalinke@yahoo.com.br
}

Resumo: O presente artigo apresenta um mapeamento sistemático (MS) realizado a partir de teses e dissertações que contemplam a Robótica Educacional como um recurso tecnológico para a ensino de conteúdos matemáticos, evidenciando uma descrição dos trabalhos associados às metodologias de ensino e teorias nelas utilizadas. O mapeamento foi realizado pelos trabalhos disponibilizados nos periódicos da Capes, no catálogo de teses e dissertações da Capes e na Biblioteca Digital Brasileira de Teses e Dissertações (BDTD), no recorte temporal de 1996 a 2020 e apresentou como resultado inicial 249 trabalhos. Após o processo de parametrização, com o estabelecimento de 7 descritores, sobraram 23 trabalhos que atendiam a temática proposta. Como resultado, constatou-se que os conceitos matemáticos se fazem presentes em atividades com uso da Robótica Educacional como um recurso tecnológico e observa-se diferentes teorias que alicerçam as pesquisas apresentadas. A Robótica Educacional é abordada junto a resolução de problemas, investigação matemática, jogos e modelagem matemática.

Palavras-chave: Ensino de Matemática. Robótica Educacional. Mapeamento Sistemático. Tecnologias Digitais. Metodologia de Ensino de Matemática.

\section{Mathematics Teaching Methodology in Educational Robotics: a systematic mapping}

\begin{abstract}
The following article presents a systematic mapping (SM) carried out based on theses and dissertations which hold educational robotics as a technological resource for the teaching of mathematical contents, providing evidence describing written papers associated to the educational methodologies and theories used therein. The mapping was obtained from articles in magazines issued by Capes, in the theses and dissertation catalogue at Capes and in the Brazilian Digital Library of Theses and Dissertations (BDTD), covering the period of 1996 to 2020, which showed an initial result of 249 articles. After setting out the parameters and establishing 7 descriptors, 23 papers covered the proposed thematic. As a result, it was established that the mathematical concepts are present in activities using educational robotics as a technological resource and that different theories give credence to the research presented. Educational robotics is broached in conjunction with problem solving, mathematical investigation, games and mathematical modelling.
\end{abstract}

Keywords: Mathematics Teaching. Educational Robotics. Systematic Mapping. Digital Technologies. Mathematics Teaching methodology.

\section{Introdução}

Mesmo com a presença de diferentes tecnologias nas escolas, que podem ser utilizadas como recursos pedagógicos, muito ainda se tem a conhecer sobre a sua inserção no ensino de Matemática. No contexto escolar, existem diversos recursos tecnológicos 
que podem ser utilizados nas aulas de Matemática, para Araújo e Mafra (2015, p. 57) “um dos recursos tecnológicos que começam a se embrenhar nos ambientes escolares brasileiros é a robótica, por esse motivo denominado Robótica Educacional".

A Robótica Educacional (RE) pode ser explorada como um recurso para o ensino de conteúdos em diferentes áreas do conhecimento, inclusive na Matemática. D'Abreu (1995) destaca que um ambiente proporcionado pelo uso da RE oportuniza uma visualização mais concreta no uso de conceitos de Matemática, Física, Programação e Engenharia na criação dos projetos robóticos. Para Campos (2019, p. 28), a busca de informações sobre a RE tem aumentado e "muitas tentativas têm sido feitas pelo mundo para introduzir o tema nas escolas, desde a educação infantil até o ensino médio". Felcher, Pinto e Folmer (2019, p. 14), apontam que a RE "se insere como uma opção com perspectivas interessantes, mas ainda não muito exploradas". Diante desse cenário, e na busca por colaborar com compreensões sobre a temática, objetiva-se nesse trabalho direcionar um olhar às possibilidades metodológicas do ensino de Matemática que são utilizadas com uso da RE.

Podemos encontrar diferentes possibilidades metodológicas voltadas para o ensino de Matemática. Para Moreira (2019) as principais são: a etnomatemática, a tecnologia digital, a modelagem matemática, a resolução de problemas, a história da matemática e a inclusão. Além destas, existem outras, como a investigação matemática e a leitura e escrita, entre outras, que são investigadas por diferentes autores e pesquisadores.

Parte-se do pressuposto que, ao utilizar a RE nas aulas de Matemática, é preciso estar atento para o encaminhamento metodológico que será dado na condução das atividades. Campos (2019) ressalta que o sucesso de uma inovação educacional não acontece pelo acesso a uma nova tecnologia, pois, por si só, ela não pode atuar de maneira direta no processo de aprendizagem. Sendo assim, como qualquer outra tecnologia aplicada à educação, a RE precisa estar atrelada às metodologias de ensino a serem utilizadas.

Nesse panorama, a inserção da RE nas aulas de Matemática gera questionamentos relacionados à metodologia a ser explorada e aos encaminhamentos propostos para a condução das atividades. Em vista disso, apresentamos como objetivo verificar, a partir de trabalhos já publicados, quais os encaminhamentos metodológicos utilizados no ensino de conteúdos matemáticos quando se utiliza a RE como um recurso pedagógico.

Assim, apresenta-se a partir de um mapeamento sistemático (MS), o que revelam as pesquisas sobre as diferentes metodologias utilizadas no ensino de conceitos matemáticos com uso da RE. A estratégia utilizada para realizar o trabalho será por meio do mapeamento das pesquisas, sua categorização e, por fim, a descrição dos resultados encontrados.

\section{Material e Métodos}

Diante do objetivo apresentado, optou-se por investigar as pesquisas que abordam a RE quanto um recurso para o ensino de conceitos matemáticos no Educação Básica, usando a metodologia de mapeamento para apresentação dos encaminhamentos metodológicos e aspectos teóricos que são revelados nos trabalhos mapeados. Sendo assim, este estudo baseou-se em Fiorentini et al. (2016), que define o mapeamento de pesquisas por "um processo sistemático de levantamento e descrição de informações acerca das pesquisas produzidas sobre um campo específico de estudo, abrangendo um determinado espaço (lugar) e período de tempo" (FIORENTINI et al., 2016, p. 18).

Para este MS foram consideradas as produções disponibilizadas em duas bases de dados: o Catálogo de Teses e Dissertações da Capes, e o Banco de Dissertações e Teses 
Digitais (BDTD). As buscas ocorreram no mês de setembro e outubro de 2020, com o uso dos operadores booleanos "AND" e "OR para organização das strings de busca, que foram assim estabelecidas: "robótica-educacional" AND ("matemática" OR "educaçãomatemática"),"robótica-pedagógica" AND ("matemática" OR "educação-matemática") e "robótica-educativa" AND ("matemática" OR "educação-matemática").

A escolha por essas strings de busca, se deu a partir das nomenclaturas (Robótica Educacional, Robótica Pedagógica e Robótica Educativa) que se mostraram mais adotadas pelos autores e pesquisadores de RE. Visando selecionar as pesquisas que abordassem os conteúdos matemáticos, realizamos uma composição com as palavras "matemática" e "educação matemática"

Foram encontradas 30 teses e 219 dissertações que compõem a totalidade de textos identificados. Em seguida aplicamos os critérios de inclusão e exclusão dos trabalhos selecionados e após a parametrização das pesquisas com foco na Educação Básica, como apresentado no fluxograma dos caminhos realizados no mapeamento (Figura 1).

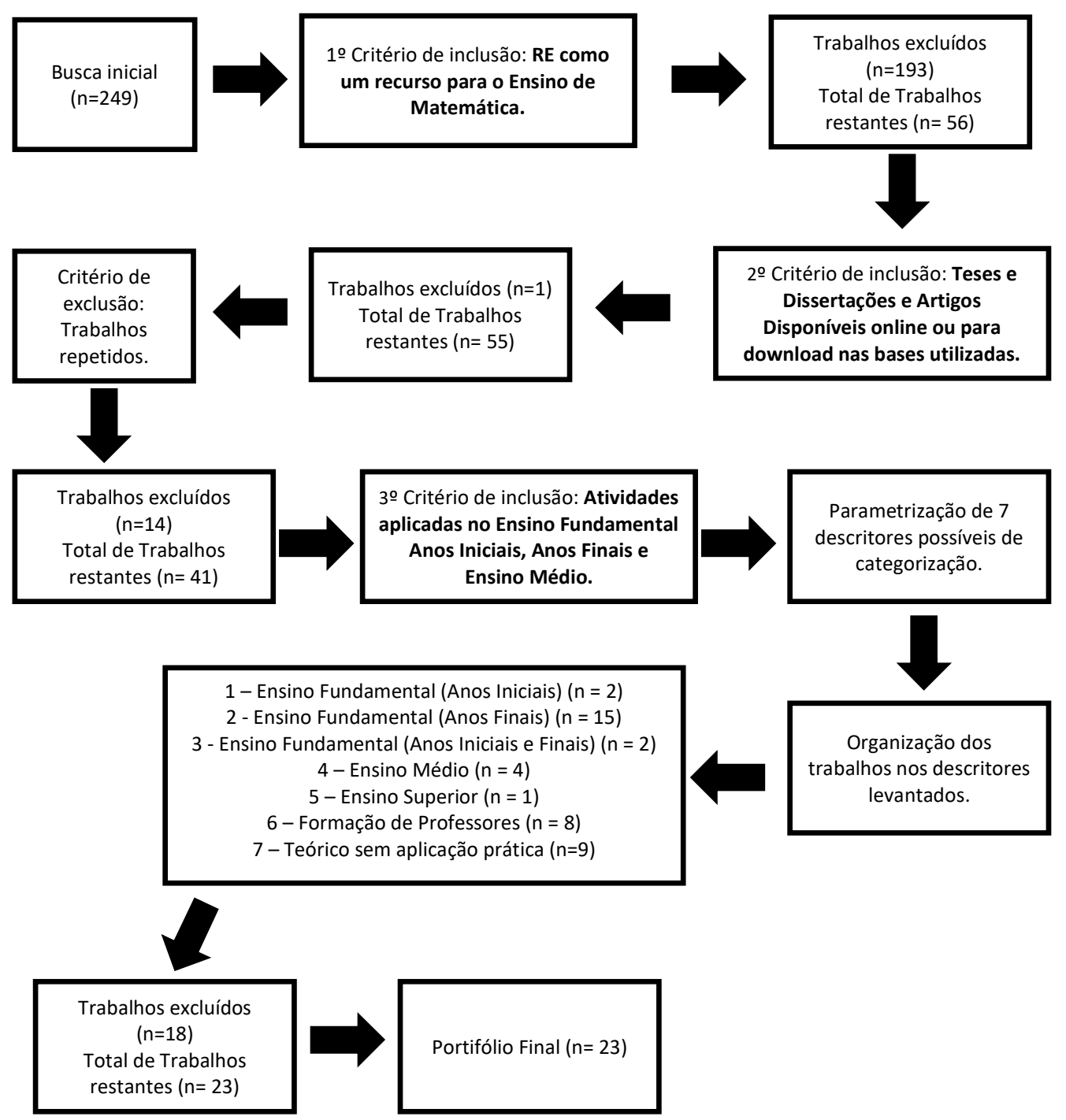

Figura 1 - Fluxograma do mapeamento realizado

Chegamos, então, a um portifólio de 23 dissertações. Com isso, realizamos a coleta de dados para a constituição da categorização. 


\section{Os trabalhos identificados}

No Quadro 1 estão relacionadas as 23 pesquisas identificadas no MS. Elas foram organizadas por título, autor e base de dados.

Quadro 1 - Identificação dos estudos primários.

\begin{tabular}{|c|c|c|c|}
\hline & Título & Autor & $\begin{array}{c}\text { Base de } \\
\text { dados }\end{array}$ \\
\hline 1 & $\begin{array}{l}\text { Robótica e as transformações geométricas: um estudo } \\
\text { exploratório com alunos do Ensino Fundamental }\end{array}$ & Accioli (2005) & BDTD \\
\hline 2 & $\begin{array}{l}\text { Robótica educacional como cenário investigativo nas aulas de } \\
\text { Matemática }\end{array}$ & Maliuk (2009) & BDTD \\
\hline 3 & $\begin{array}{l}\text { Exploração de tópicos de Matemática em modelos robóticos: } \\
\text { Com utilização do software SLOGO no Ensino Médio }\end{array}$ & Furletti (2010) & CAPES \\
\hline 4 & $\begin{array}{c}\text { A dança dos robôs: qual a Matemática que emerge durante uma } \\
\text { atividade lúdica com Robótica Educacional? }\end{array}$ & Leitão (2010) & CAPES \\
\hline 5 & $\begin{array}{l}\text { Robótica educacional: socializando e produzindo conhecimentos } \\
\text { matemáticos }\end{array}$ & Moraes (2010) & BDTD \\
\hline 6 & $\begin{array}{l}\text { Uso da Robótica no ensino de proporção aos alunos do Ensino } \\
\text { Fundamental II }\end{array}$ & $\begin{array}{l}\text { Nascimento } \\
(2012)\end{array}$ & CAPES \\
\hline 7 & $\begin{array}{l}\text { Robótica na sala de aula de Matemática: os estudantes aprendem } \\
\text { matemática? }\end{array}$ & Martins (2012) & BDTD \\
\hline 8 & $\begin{array}{c}\text { O uso da Robótica Educativa e o desenvolvimento de } \\
\text { competências e habilidades matemáticas }\end{array}$ & $\begin{array}{l}\text { Almeida Neto } \\
(2014)\end{array}$ & CAPES \\
\hline 9 & $\begin{array}{l}\text { A Robótica Educacional como meio para a aprendizagem da } \\
\text { Matemática no Ensino Fundamental }\end{array}$ & Gomes (2014) & CAPES \\
\hline 10 & $\begin{array}{l}\text { A robótica como auxílio à aprendizagem da Matemática: } \\
\text { percepções de uma professora do Ensino Fundamental público }\end{array}$ & Rodarte (2014) & CAPES \\
\hline 11 & $\begin{array}{l}\text { Robótica Educacional e raciocínio proporcional: uma discussão à } \\
\text { luz da teoria da relação com o saber }\end{array}$ & $\begin{array}{l}\text { Oliveira } \\
(2015)\end{array}$ & CAPES \\
\hline 12 & $\begin{array}{c}\text { Atividades com Robótica Educacional para as aulas de } \\
\text { Matemática do } 6^{\circ} \text { ao } 9^{\circ} \text { ano do Ensino Fundamental: utilização } \\
\text { da metodologia LEGO } \AA \text { Zoom Education }\end{array}$ & $\begin{array}{l}\text { Rodrigues } \\
\quad(2015)\end{array}$ & CAPES \\
\hline 13 & $\begin{array}{l}\text { Robótica Educativa: um recurso para o estudo de geometria plana } \\
\text { no } 9^{\circ} \text { ano do Ensino fundamental }\end{array}$ & $\begin{array}{l}\text { Wildner } \\
(2015)\end{array}$ & CAPES \\
\hline 14 & $\begin{array}{l}\text { Ensino das Relações Métricas do Triângulo Retângulo com } \\
\text { Robótica Educacional. }\end{array}$ & Santos (2016) & CAPES \\
\hline 15 & $\begin{array}{c}\text { Função polinomial do } 2^{\circ} \text { grau: uma sequência didática apoiada } \\
\text { nas tecnologias digitais }\end{array}$ & $\begin{array}{c}\text { Casagrande } \\
(2017)\end{array}$ & CAPES \\
\hline 16 & $\begin{array}{l}\text { Robótica nas aulas de Matemática: Uma perspectiva tecnológica } \\
\text { associada ao ensino de funções }\end{array}$ & $\begin{array}{c}\text { Oliveira } \\
(2017)\end{array}$ & CAPES \\
\hline 17 & $\begin{array}{l}\text { Uma aplicação da Robótica Educacional no estudo do } \\
\text { número irracional } \pi \text { utilizando LEGO MINDSTORM EV3 }\end{array}$ & Armão (2018) & CAPES \\
\hline 18 & $\begin{array}{l}\text { Robótica Educacional e o ensino de Matemática: um } \\
\text { experimento educacional em desenvolvimento no Ensino } \\
\text { Fundamental }\end{array}$ & Galvão (2018) & CAPES \\
\hline 19 & $\begin{array}{c}\text { Inserção da Robótica Educacional nas aulas de Matemática: } \\
\text { desafios e possibilidades }\end{array}$ & $\begin{array}{l}\text { Maffi } \\
(2018)\end{array}$ & CAPES \\
\hline 20 & Robótica educativa na construção do pensamento matemático. & Aragão (2019) & BDTD \\
\hline 21 & Matemática com tecnologias: cubo de Rubik e robótica. & $\begin{array}{c}\text { Barbosa } \\
(2019)\end{array}$ & CAPES \\
\hline 22 & $\begin{array}{l}\text { Assimilação de conceitos relacionados a triângulos e } \\
\text { quadriláteros através da Robótica Educativa }\end{array}$ & $\begin{array}{l}\text { Rüdell } \\
(2019)\end{array}$ & CAPES \\
\hline 23 & $\begin{array}{l}\text { Robótica Educacional no ensino fundamental I: perspectivas e } \\
\text { práticas voltadas para a aprendizagem da matemática. }\end{array}$ & $\begin{array}{l}\text { Zilio } \\
(2020)\end{array}$ & BDTD \\
\hline
\end{tabular}


Durante a leitura dos trabalhos notamos que a geometria está presente em 64\% deles. No Gráfico 1 estão apresentadas as unidades temáticas que foram identificadas.

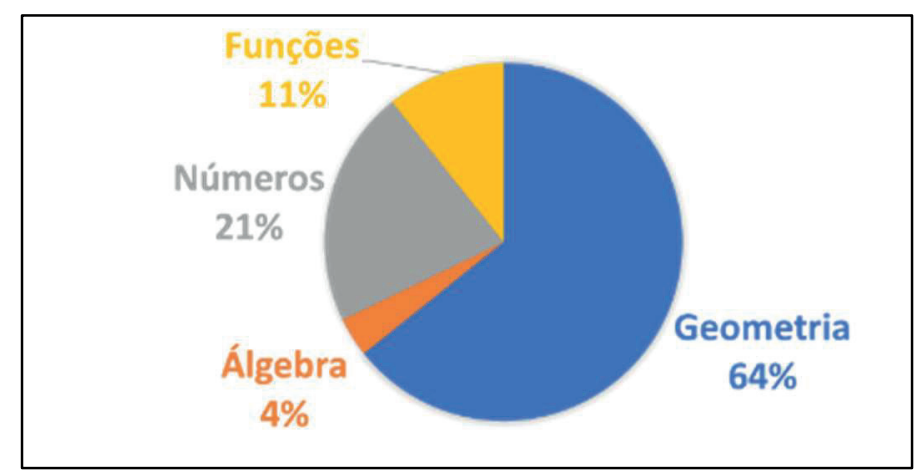

Gráfico 1 - Conteúdos matemáticos identificados nos trabalhos

Para categorizar dos dados coletados adotou-se as ideias de Fiorentini e Lorenzato (2006), para quem a categorização "significa um processo de classificação ou de organização de informações em categorias, isto é, em classes ou conjuntos que contenham elementos ou características comuns" (FIORENTINI; LORENZATO, 2006, p. 134).

Na Figura 2, expomos uma nuvem de palavras com os principais termos presentes na metodologia dos trabalhos de cada uma das pesquisas. A intenção era observar quais palavras que remetem às possibilidades metodológicas do ensino de matemática. Foram elas: resolução de problemas, investigação, modelagem e jogos. Já em relação aos aspectos teóricos observou-se que construcionismo de Papert e construtivismo de Piaget se revelaram presentes.

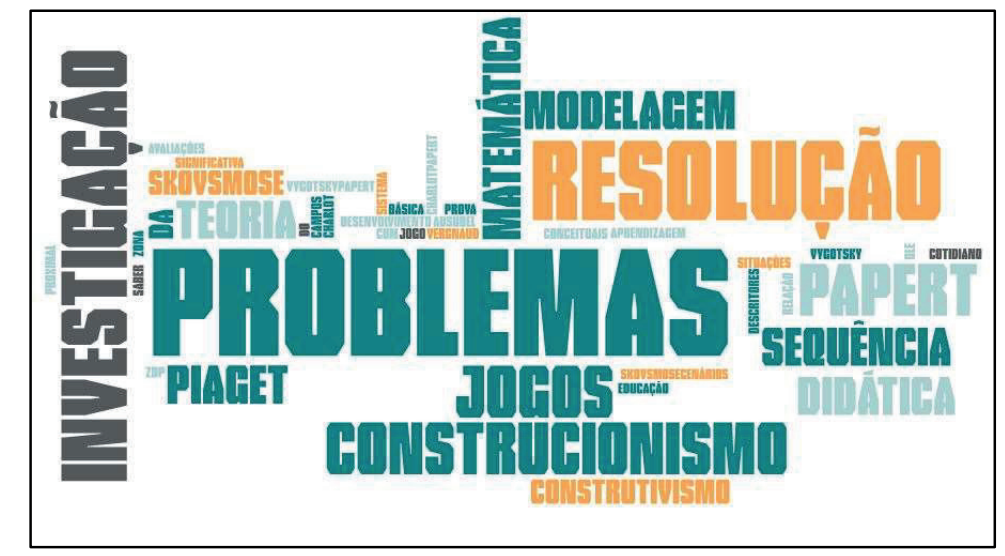

Figura 2 - Nuvem de palavras

Para dispor as categorias, vamos seguir a proposta de Fiorentini (2002) utilizando focos e subfocos temáticos. Estabelecemos as possibilidades metodológicas e os aspectos teóricos como focos temáticos, e como subfocos suas ramificações. Os subfocos identificados em relação às possibilidades e elencados na categorização são: resolução de problemas, investigação matemática, jogos e modelagem matemática.

Em relação aos aspectos teóricos, emergiram as seguintes subfocos: situações do cotidiano e teoria dos campos conceituais de Vergnaud (1997), teoria de aprendizagem significativa Ausubel (1980), construtivismo de Piaget, construcionismo de Papert, zona de desenvolvimento proximal de Vygotsky (2007), teoria da relação com saber de Charlot (2005). Convém mencionar que um trabalho pode pertencer a mais de um foco ou subfoco e que a organização, presente na Figura 3, mostra a percepção dos autores deste artigo. 


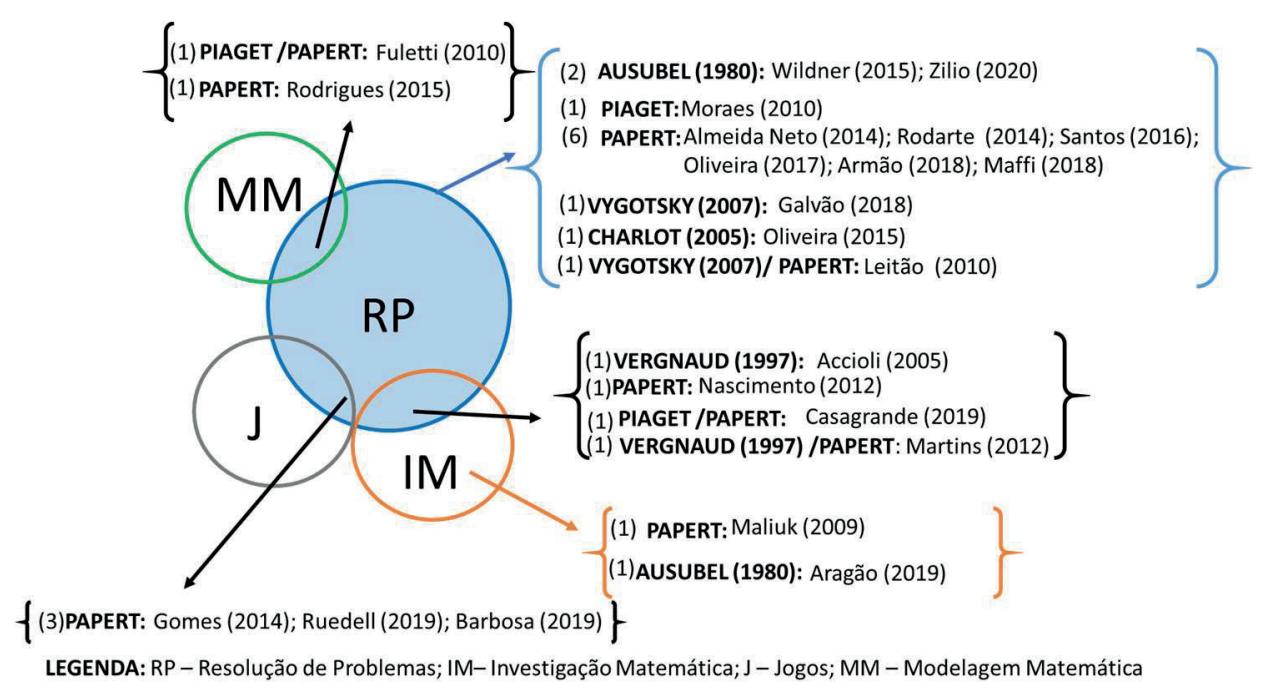

Figura 3 - Focos e subfocos das pesquisas trabalhos

A partir da categorização das pesquisas, apresentamos uma descrição dos

\section{Discussão dos resultados}

Direcionamos a condução do portifólio bibliográfico com base nos focos e subfocos apresentados anteriormente, assim apresentamos as seis categorias que emergiram desta pesquisa: (1) "resolução de problemas", (2) "resolução de problemas e investigação matemática", (3) "investigação matemática", (4) "resolução de problemas e modelagem matemática" e (5) "resolução de problemas e jogos".

Iniciamos pela categoria de "resolução de problemas", a qual engloba a maioria das pesquisas, e assim, se revela um tópico a ser fortemente considerado, pois indica o procedimento metodológico mais utilizado nas aulas que fazem o uso da RE enquanto recurso para o ensino de conceitos matemáticos. Nestas pesquisas, as atividades são propostas no formato de sequências didáticas com foco nos conteúdos curriculares e utilizam o modelo robótico para relacionar a teoria e a prática. Como exemplo, Almeida Neto (2014) descreveu sua experiência com turmas do Ensino Fundamental Anos Finais, abordando os conteúdos matemáticos vistos em sala de aula a partir de montagens e programações dos robôs. Nas atividades propostas e realizadas o autor relacionou os conteúdos matemáticos com os descritores da matriz referencial da Prova Brasil de Matemática, destacando que usou 15 dos 37 descritores da matriz de referência do Sistema de Avaliação da Educação Básica para Prova Brasil do $9^{\circ}$ ano do Ensino Fundamental.

Ainda nesse contexto, aponta-se como exemplo a dissertação de Armão (2018) que direciona o uso da RE como ferramenta metodológica para o estudo do número irracional $\pi$ nas aulas de Matemática. A proposta foi alinhada com a Base Nacional Comum Curricular e teve como público estudantes do Ensino Fundamental Anos Finais de uma escola particular. Para isso, o autor relatou a elaboração de quatro planos de aulas que são: Plano de Aula A: Conceitos Preliminares; Plano de Aula B: Descobrindo o $\pi$ com a Robótica; Plano de Aula C: Um desafio de robótica e Plano de Aula D: Formalizando conceitos e praticando.

Em seis dos trabalhos dentro da categoria de "resolução de problemas", o aspecto teórico mais utilizado foi o construcionismo de Papert (2008). Este fato, pode se dar devido as possibilidades de contextualização da RE nas aulas de Matemática, bem como oportunizar um ambiente diferenciado de ensino, possibilitando discussões, colocando o 
estudante como condutor no processo da construção do seu próprio conhecimento e trocas de saberes.

Nesse sentido, Maffi (2018, p.73) ressalta que a RE pode contribuir para oportunizar aos "estudantes o reconhecimento de que os conhecimentos matemáticos são essenciais para o entendimento, compreensão e atuação no mundo". Corroborando com isso, ao utilizar a resolução de problemas a partir de um padrão pré-estabelecido, pode ocasionar "a mudança de foco do pensar se as próprias regras são eficazes na aplicação imediata, para procurar explicações múltiplas de modo como trabalhar com as regras pode contribuir, a longo prazo, para a aprendizagem" (PAPERT, 2008, p. 91). A referir-se ao uso de regras, Papert (2008) aponta que a resolução de problemas matemáticos pode ser apresentada pelos professores de uma forma diferenciada, pois algumas vezes não condizem com a realidade, ou não possibilitam o desenvolvimento do pensamento matemático por meio de ofertar um ambiente diferenciado de ensino.

A segunda categoria "resolução de problemas e investigação matemática", indica que as possibilidades metodológicas podem ser trabalhadas em uma mesma atividade. Neste sentido, Accioli (2005) apresentou um estudo exploratório a partir de uma investigação sobre o conteúdo de simetria e utilizou como base teórica Vergnaud (1997), na perspectiva das relações do cotidiano. Ainda neste cenário, Casagrande (2017) com base nas ideias do contrucionismo de Papert e Construtivismo de Piaget destacou que por meio da resolução de problemas e da investigação matemática a RE pode ser usada para contextualizar e promover experiências práticas em relação aos conteúdos matemáticos. Já Martins (2012) usou como base teórica o construtivismo de Papert (2008) para abordar o uso de tecnologias na escola e discutiu a aprendizagem de conceitos matemáticos à luz da Teoria dos Campos Conceituais de Vergnaud.

O uso da "investigação matemática", categoria que emergiu nas pesquisas de Maliuk (2009) e Aragão (2019), se destaca pela criação dos cenários investigativos. Neste prisma, apresentamos como exemplo a pesquisa de Maliuk (2009), que utilizou a RE situando-a na abordagem proposta por Ole Skovsmose chamada de cenários para investigação, que são estruturados para acontecer em paralelo com a sala de aula tradicional. A autora relatou a montagem de um carrinho com dois motores independentes que possibilitou o estudo do movimento e de rotação dos ângulos, concluindo que as atividades com robótica se mostraram apropriadas para explorar, desenvolver e aprofundar conceitos matemáticos. Além disso, para os autores essa metodologia apresenta o professor como mediador do ensino, possibilitando momentos de investigação criação de conceitos matemáticos no processo de aprendizagem dos estudantes.

$\mathrm{Na}$ quarta categoria "resolução de problemas e modelagem matemática", os autores apontam a criação de modelos matemáticos, advindos das práticas realizadas nas atividades propostas. Furletti (2010) descreveu a construção de um dispositivo robótico programado em ambiente LOGO como ferramenta exploratória de ensino de conceitos matemáticos. $\mathrm{O}$ autor propôs uma sequência didática, que iniciou com a construção de uma roda gigante e depois encaminhou para a programação deste protótipo. Na sequência o pesquisador relatou uma proposta de resolução de problemas, concluindo que a utilização da roda gigante com foco no estudo de funções tinha como objetivo formular modelos matemáticos. Nessa premissa, Rodrigues (2015), por sua vez, destacou em sua pesquisa a proposta de modelar situações problemas por meio de equações e sistemas de equações do primeiro grau, revisar fração e proporção e interpretar, converter e realizar operações com unidades de medidas.

O uso de "jogos" junto a "resolução de problemas" foi outra categoria que emergiu das pesquisas encontradas. Nas aulas de Matemática com o apoio da RE essa 
categoria fez parte do trabalho de Barbosa (2019), em que a proposta foi resolver o cubo de Rubik por meio da RE e associar essa experiência com a resolução de problemas matemáticos aulas extracurriculares. Nesta proposta Gomes (2014), apresentou uma sequência didática na qual foram explorados conceitos de geometria plana e destacou que uma das vantagens dos usos de jogos junto as práticas visando o ensino de matemática é a utilização das "técnicas de resolução de problemas como forma de investigar e solucionar os desafios propostos" (GOMES, 2014, p. 60).

Wildner (2011) e Zilo (2020) apresentam como teoria norteadora para as pesquisas, a teoria da aprendizagem significativa de Ausubel, ressaltando que o papel do estudante como autônomo na criação e projetos e na interação entre a teoria e prática, visando um maior significado para o contexto voltado para a sociedade. A vista disso, Zilo (2020) relata que tal teoria proposta por Ausubel junto ao uso da RE, assume uma relação entre os saberes pré-existentes e os novos, tal como contribui para a contextualização com a realidade, "proporcionando novos horizontes e saberes" (ZILO, 2020, p. 28).

Já em relação a teoria da relação do saber de Charlot, Oliveira (2015) ressalta a importância das relações estabelecidas dos estudantes com o mundo, com o outro e consigo mesmo para o processo de aprendizagem. Além disso, Galvão (2018) ao considerar a teoria de aprendizagem de Vygotsky, destaca que a tecnologia começa a fazer parte do cotidiano dos indivíduos, de tal forma a possibilitar a reorganização do pensamento e que RE com a criação e montagem de protótipos junto ao uso das tecnologias digitais para automatizar esse modelo pode contribuir para esse processo de reorganização.

As dissertações apresentadas revelaram aspectos que envolvem a prática com a $\mathrm{RE}$ ao proporcionar o ensino de conceitos matemáticos, bem como suas estratégias metodológicas, teorias e conteúdos relacionados com a simulações, montagem de protótipos e programações que são propostas ou podem emergir durante a realização da atividade.

Percebeu-se que a resolução de problemas foi a possibilidade metodológica mais utilizada e em relação aos aspectos teóricos o mais referenciado nas pesquisas foi $\mathrm{o}$ construcionismo de Papert. Na sua totalidade os resultados convergem para a resolução de problemas matemáticos, com uso da criação e programação de protótipos robóticos. Isso indica que esse recurso se faz presente, de alguma forma, no ensino ou na aprendizagem de conceitos matemáticos com a RE.

Além da resolução de problemas, a investigação matemática se apresentou em 6 pesquisas. Esse fato, pode ser entendido a partir das ideias de Ponte, Brocado e Oliveira (2013, p. 16) de que "uma investigação se desenvolve usualmente em torno de um ou mais problemas", tal como "o grande passo de uma investigação é identificar claramente o problema a resolver". Nessa perspectiva, a RE pode ser entendida como um recurso para contextualização, resolução e formulação de problemas, estratégias de investigação, tomadas de decisões, formulação e aplicação de soluções, entre outras possibilidades.

\section{Considerações}

O MS aqui apresentado resultou em 23 trabalhos. Para identificar as informações que possibilitassem a categorização dos estudos, realizamos a leitura dos resumos com o intuito de identificar os dados relevantes para esse estudo. Percebemos que alguns trabalhos, em seus resumos, não apresentavam as informações necessárias para esta pesquisa, o que necessitou uma busca mais criteriosa na leitura integral dos textos. A partir disso, buscamos identificar informações que possibilitassem a categorização desta pesquisa. 
Ao tecer a categorização, separamos em quatro categorias. Destacamos que os dois focos se completaram, pois um deles abordou as possibilidades metodológicas que são utilizadas nas atividades de RE e outro fez referência as teorias e aportes teóricos citados nos trabalhos.

A questão levantada no MS foi respondida com os passos apresentados no mapeamento das pesquisas. Desta forma, foi possível identificar que a RE vem sendo utilizada com a proposta da resolução de problemas, investigação matemática, jogos e modelagem. Estas podem ser relacionar entre si em atividades contextualizadas envolvendo relações entre a teoria e a prática com o uso de conceitos matemáticos.

O foco na resolução de problemas foi contemplado em 21 dissertações. Com isso, percebemos que existe uma preocupação em relação à contextualização das atividades propostas e amparadas nas teorias utilizadas. Além disso, foi possível identificar também que em muitas vezes elas se completam e no decorrer das atividades são evidenciadas.

Para tanto, buscando apresentar como a RE vem sendo utilizada, quando proposta para o ensino de conceitos matemáticos na Educação Básica, o MS revelou que estratégias metodológicas são incorporadas nas práticas e que os aspectos teóricos são importantes para elaboração e condução das atividades. Assim, este estudo revelou que ao aproveitar situações-problema que a RE oferece e ao utilizar a Matemática para buscar soluções, pode assim surgir uma estratégia que direciona os encaminhamentos metodológicos para uso deste recurso.

Com esse estudo, percebemos que as práticas que fazem o uso da RE visando o ensino de conceitos matemáticos, indicam a estruturação de encaminhamentos metodológicos por parte dos professores, que por sua vez utilizam de fundamentações teóricas. Isso vai ao encontro com papel do professor nas atividades que usam a RE nas aulas de matemática, se apresentando como de mediador do processo de construção dos conceitos matemáticos. Cabendo a ele direcionar a criação de cenários, para que os estudantes possam explorar as montagens, a programação, bem como as estratégias de solucionar os problemas e desafios propostos.

Para finalizar, esperamos que esse mapeamento possa contribuir para futuros estudos relacionados a temática apresentada. Deste modo, diante do cenário atual, fica a inspiração para a apresentação de pesquisas direcionadas ao uso da RE e sua relação com os conceitos matemáticos, visto que no contexto escolar a matemática pode estar inserida junto a essa tecnologia, ressaltando assim a importância de trabalhos sobre o uso desse recurso, visando apresentar práticas voltadas para a construção de saberes matemáticos.

\section{Referências}

ACCIOLI, R. M. (2005). Robótica e as transformações geométricas: um estudo exploratório com alunos do ensino fundamental, no Estado de São Paulo. São Paulo: Educação Matemática/PUC, 224p. Dissertação de Mestrado.

ALMEIDA NETO, C. F. de. (2014). O uso da robótica educativa e o desenvolvimento de competências e habilidades matemáticas, no Estado do Ceará. Juazeiro do Norte: PROFMAT/UFC, 106p. Dissertação de Mestrado.

ARAUJO, C. A. P.; MAFRA, J. R. e S. (2015). Robótica Educacional: ensaios teóricos e práticas experimentais. Curitiba: $\mathrm{CRV}, 156 \mathrm{p}$.

BARBOSA, C. M. (2019). Matemática com tecnologias: cubo de Rubik e robótica, no Estado de Goiás. Goiás: Unidade Acadêmica Especial de Matemática e Tecnologia/UFG, 101p. Dissertação de Mestrado.

CASAGRANDE, E. (2017). Função polinomial do $2^{\mathbf{0}}$ grau: uma sequência didática apoiada nas tecnologias digitais, no Estado do Rio Grande do Sul. Passo Fundo: Instituto de Ciências Exatas e Geociências/UPF, 135 p. Dissertação de Mestrado. 
CAMPOS, F.R. (2019). A Robótica para uso Educacional. Editora Senac São Paulo. São Paulo - SP, 208p.

D' ABREU, J. V. V. (1995). Design de dispositivos: uma abordagem interdisciplinar. VII Congresso Internacional Logo / I Congresso de Informática Educativa do Mercosul. Porto Alegre, UFRS, p. 48 - 55.

FELCHER, C. D. O.; PINTO, A. C. M. P.; FOLMER, V. (2019). Tendências em Tecnologias digitais no Ensino da Matemática Reveladas no EBRAPEM. Revista Educação Matemática Pesquisa, São Paulo, v. 21, n. 2, p.1-22. Disponível em: $<$ https://revistas.pucsp.br/emp/article/view/39080>. Acesso em: 15 set. 2020.

FURLETTI, Saulo. (2010). Exploração de tópicos de Matemática em modelos robóticos com utilização de software SLOGO no Ensino Médio, no Estado de Minas Gerais. Belo Horizonte: Ensino de Ciências e Matemática/PUC, 134p. Dissertação de Mestrado.

GALVÃO, A.P. (2018). Robótica Educacional e o Ensino de Matemática: um experimento educacional em desenvolvimento do ensino fundamental, no Estado do Pará. Santarém: Instituto de Ciências da Educação/UFOPA, 135p. Dissertação de Mestrado.

GOMES, P. N. N. (2014). A Robótica Educacional como meio para a Aprendizagem da Matemática no Ensino Fundamental, no Estado de Minas Gerais. Lavras: Programa de Pós-Graduação em Educação/UFLA, 95p. Dissertação de Mestrado.

MAFFI, C. (2018). Inserção da Robótica Educacional nas aulas de matemática: desafios e possibilidades, no Estado do Rio Grande do Sul. Porto Alegre: Escola de Ciências/PUC, 108 p. Dissertação de Mestrado.

MALIUK, K. D. (2009). Robótica Educacional como cenário investigativo nas aulas de matemática, no Estado do Rio Grande do Sul. Porto Alegre: Instituto de Matemática/UFURS, 91p. Dissertação de Mestrado.

MARTINS, E. F. (2012). Robótica na sala de aula de matemática: os estudantes aprendem matemática?, no Estado do Rio Grande do Sul. Porto Alegue: Mestrado Profissional em Ensino de Matemática/Instituto de Matemática, 168p. Dissertação de Mestrado.

MOREIRA, E. C. (2019). Tendências em educação matemática com enfoque na atualidade. In: NEVES, da S. P. N.; DÖRR, R. C. (Ed.). Formação de professores de matemática desafios e perspectivas. Curitiba: Appris, p. 45-64.

OLIVEIRA, E. S. de. (2015). Robótica Educacional e raciocínio proporcional: uma discussão à luz da teoria da relação com o saber, no Estado de Paraíba. Campina Grande: Centro de Ciências e Tecnologia/ UFPA, 157p. Dissertação de Mestrado.

PAPERT, S. (2008). A Máquina das Crianças: repensando a escola na era da informática. Tradução Sandra Costa. Porto Alegre: ARTMED, 220p.

RODRIGUES, W. S. (2015). Atividades com Robótica Educacional para as aulas de matemática do $6^{\circ}$ ao $9^{\circ}$ Ano do Ensino Fundamental: utilização da metodologia LEGO Zoom Education, no Estado de São Paulo. Ilha Solteira: Instituto de Biociências, Letras e Ciências Exatas, Universidade Estadual Paulista Júlio Mesquita Filho, 106 p. Dissertação de Mestrado.

WILDNER, M.C.S. (2015). Robótica Educativa: um recurso para o estudo de Geometria Plana no $9^{\circ}$ ano do Ensino Fundamental, no Estado do Rio Grande do Sul. Lajeado: Ensino de Ciências Exatas/UNIVATES, 155 p. Dissertação de Mestrado.

ZILIO, C. (2020). Robótica Educacional no ensino fundamental I: perspectivas e práticas voltadas para a aprendizagem da matemática, no Estado do Rio Grande do Sul. Porto Alegre: Educação em Ciências: Química da Vida e Saúde/FURG, 53 p. Dissertação de Mestrado. 